\title{
AlnC: An extensive database of long non-coding RNAs in
}

\section{Angiosperms}

Ajeet Singh ${ }^{1}$, AT Vivek ${ }^{1}$, Shailesh Kumar $^{1 *}$

4

\section{*Corresponding author}

Shailesh Kumar

https://orcid.org/0000-0002-1872-9903

${ }^{1}$ Bioinformatics Lab, National Institute of Plant Genome Research (NIPGR), Aruna Asaf Ali Marg, New Delhi 110067, India. Tel: 91-11-26735217, Fax: 91-11-26741658, Email: shailesh@nipgr.ac.in

\section{Abstract}

Long non-coding RNAs (lncRNAs) play a major role in diverse biological processes that are contemplated to have diverse regulatory roles in plants. While lncRNAs have been described and annotated in major plant species, many of plant species lack annotated lncRNAs despite the availability of transcriptome data in public repositories. In this study, we have identified and annotated the lncRNAs in the transcriptomic data from the 1000 plant genome project (1KP), and developed a user-friendly, open-access database called AlnC. This can be used for the exploration of lncRNAs in 682 Angiosperm plants. The current version of AlnC offers 10,855,598 annotated lncRNA transcripts across 809 sample tissues of diverse flowering plants. To enhance the AlnC interface, we have provided the features for browsing, searching and downloading of lncRNA data, interactive graphics, and online BLAST service. In addition to these information, each lncRNA record is annotated with possible Short open reading frames (e.g. sORFs) to facilitate the study of peptides encoded within lncRNAs. With this user-friendly interface, we assume that AlnC will serve as a rich source of lncRNAs that will contribute to small-and large-scale studies in a wide range of flowering plants.

Keywords: Angiosperms; 1KP Project; lncRNAs; RNA-seq; sORFs

Database URL: http://nipgr.ac.in/AlnC 


\section{Introduction}

Angiosperms (flowering plants) are land plants with more than 3,000,000 recorded species worldwide, comprising one of the most diverse group within the plant kingdom [1,2]. Most plants belonging to this group have been intensively studied in order to understand both the flowering and other major mechanisms. As a result, research in flowering plants has exploded with the advent of next generation sequencing leading to an improved picture of transcriptome, especially from the point of non-coding RNAs (ncRNAs). Of all recent studies in plant non-coding RNAs, long noncoding RNAs (lncRNAs) have been shown greater interest to study alongside miRNAs, which are typically more than 200 nucleotides with almost no protein coding capacity. There is also compelling evidence to lncRNAs functioning in multiple biochemical pathways of plants over the years [3-5]. Despite being in the spotlight, lncRNAs are yet to be annotated in a variety of plant species, despite the presence of data banks focused primarily on model plants and major crops. This research gap can be addressed by the use of RNA sequencing (RNA-seq) data as it provides enormous opportunities to understand transcriptome and classify potential lncRNAs $[6,7]$. Eventually, with the rise of transcriptome data in public repositories, thousands of plant lncRNAs were identified and maintained in a number of databases e.g. PLNlncRbase, CANTATAdb, GREENC, PLncDB v2.0, and lncRNAdb v2.0 [8-12]. The available lncRNA databases are limited to model plants and fewer other plants are another major concern, as information on the potential lncRNAs of many Angiosperms is still largely scarce and hinders the advancement of lncRNA research in several of these plants. Several plant species have been investigated for genome-wide lncRNAs using independent bioinformatics pipelines for annotation and archiving in databases, but several plants are still unexplored due to a complete lack of genome details. In order to address this problem in organisms with no genome data, various lncRNA methodologies are available, and are increasingly developed to improve lncRNA identification and annotation from de novo assembled transcripts $[13,14]$. 
In this current genomic era, it is essential to make lncRNA annotation models for diverse plant species in terms of improving our understanding of lncRNA biology. In parallel, the creation and maintenance of a stable lncRNA data repository is equally necessary if lncRNA biology is to be understood. $[15,16]$. In this research work, we have used large-scale transcriptome data to identify potential lncRNAs with three major goals. First, we anticipate to provide information on most potential lncRNAs of plant species with no available genome sequence. The second goal is to mediate the importance of unused large-scale datasets as an annotation source for thousands of IncRNAs. Lastly, to develop a database to act as a catalyst to promote lncRNA investigations in Angiosperms, and to provide a one-stop data access platform for lncRNA researchers. We therefore capitalised on large-scale transcriptomic data of 682 angiosperms from $1 \mathrm{Kp}$ as this enabled us to annotate 10,855,598 lncRNAs. The results of the study have been organised and deposited in a userfriendly web-interface, the Angiosperm LncRNA Catalog (AlnC) with a plan to update periodically on the basis of new knowledge and an expansion in the number of species of Angiosperm in the future.

\section{Material and Methods}

\section{Data collection and systematic IncRNA identification}

We have downloaded the de novo assembled transcripts of 682 plant species belonging to Angiosperms from $1 \mathrm{kp}$ project (http://www.onekp.com/public data.html), and identified the potential lncRNAs [17]. For each species, lncRNAs were identified from each library relying on a bioinformatics pipeline previously exploited by Singh et al., 2017, and transcripts longer than 200 bp were retained that are not overlapping with protein-coding gene models (Fig 1(A))[18]. First, we excluded potential coding transcripts (translated proteins of matched orthogroups derived from annotated plant genomes) from assembled transcripts set for that species [19]. Further, proteincoding transcripts were discarded by using PLncPRO (python prediction.py -p plncpro-result-file -i sequence.fa-m models/<monocot or dicot $>$.model -o plncpro-out $-\mathrm{d}$ lib/blastdb/swiss-protDB -t 15 -

r) on the basis of a BLAST approach with the curated list of Swiss-Prot proteins [20]. In the final 
filtering step, high-confident lncRNA transcripts were extracted by setting a minimum length threshold of $200 \mathrm{nt}$, and a non-coding probability score of 0.8 (python predstoseq.py $-\mathrm{f}$ sequence.fa -o output-file -p plncpro-result-file -1 0 -s 0.8 --min 200).

Fig 1(A) Systematic workflow adopted to annotate potential lncRNAs of all flowering plants available from the $1 \mathrm{KP}$ project.

\section{Database construction and implementation}

After the compilation of all the information regarding lncRNAs, AlnC web interface was developed using Hypertext Mark-up Language (HTML), Cascading Style Sheets (CSS), Structured Query Language, Java scripting language, PERL and Hypertext Preprocessor on Apache Hypertext Transfer Protocol server. All lncRNAs available at AlnC, and other related annotations have been handled by a relational database set up with MySQL. A stand-alone BLAST (v2.11.0) tool is installed for online similarity search feature. ViennaRNA (v2.4.16) and ORFfinder (v0.4.3) tools are used for the secondary structure visualization, and identification of Small open reading frames (sORFs) respectively [21-23].

\section{Results and Discussion}

\section{Data content in AlnC}

We have identified a total of 10,855,598 lncRNAs from 809 RNA-seq samples of Angiosperms, available at $1 \mathrm{kp}$ project webpage (http://www.onekp.com/public data.html). All the related numbers of total transcripts, filtered transcripts, lncRNAs, and filtered lncRNAs identified in each plant species are mentioned at the 'Statistics' section (http://nipgr.ac.in/AlnC/stat-data.php) of AlnC webpage. At AlnC, we have organised and compiled this catalogue of 10,855,598 lncRNAs from 809 samples available in the $1 \mathrm{KP}$ project, which functions as a comprehensive catalogue of potential lncRNAs in 682 flowering plants. No other data repositories on lncRNAs on this scale exist, and most lncRNAs of the species included in AlnC belong to poorly studied taxa, rendering AlnC of wide interest among plant researchers (Fig 1(B \& C)). At AlnC, the length of newly identified lncRNAs of flowering plants across clades have the size ranges from 200 to $7633 \mathrm{nt}$ with an average length of $405 \mathrm{nt}$ (Fig 1(C)). The median length of the identified lncRNAs is smaller than 
the median length of the coding sequences (Fig 1(B) and 3(E)). Moreover, most lncRNAs (66\%)

were less than $400 \mathrm{bp}$ in length whereas only $2.9 \%$ lncRNAs were more than $1000 \mathrm{bp}$ in length. Our attempts to identify ortholog relationships using BLASTN search reported large hits, however, we could only identify a moderate number of annotated $\operatorname{lncRNAs}$ at AlnC, identical to those stored in other databases reasoning to less conservation and apparent differences in the number of lncRNAs, but it also suggest certain significant sequence similarities across species. We have included the significant hits to NONCODE lncRNAs (384) and experimentally validated lncRNAs available in Plncdb (6) in Table S1 [11,24]. Further, the AU content of lncRNAs, available at AlnC, varied from $50-90 \%$ with an average of $75 \%$ in comparison to the coding transcripts which ranged $30-80 \%$ with an average of $60 \%$ (Fig 1(D)). Most lncRNAs contained more than $75 \%$ AU content, and the analysis implies the richness of AU than that in coding sequences [25-27]. A brief description of

119 the lncRNA entries available at $\mathrm{AlnC}$ is represented in the Fig 1 and Table 1. The relationship analysis of annotated lncRNA with assembled transcripts and protein-coding transcripts yielded two distinct clusters of monocot and dicots, respectively (Fig 2). These clusters indicates the proportional discovery rate of 1 ncRNAs with respect to the $1 \mathrm{kp}$ assembled transcripts, and also suggests the ratio of total transcripts to coding transcripts is smaller in dicot species whereas the reverse in case of monocots.

Fig 1. (B) Length-wise distribution of lncRNAs across clades, (C) Pie chart represents the percentage of lncRNA entries in AlnC, (D) Percentage composition of AU content in proteincoding transcripts and lncRNAs in AlnC.

Fig 2. Ternary plot of AlnC IncRNAs, protein-coding and $1 \mathrm{Kp}$ assembled transcripts across clades. The bubble size represents the size of $1 \mathrm{Kp}$ assembled transcripts, protein-coding and AlnC lncRNAs in (A), (B) and (C), respectively.

\section{Modules available at AlnC}

134 Current release of AlnC provides two search interfaces; (a) Simple Search, and (b) Advanced 135 Search (Fig 3(A)). 'Simple Search' allows the user to quickly search the lncRNAs on the basis of 136 taxonomic rank (Clade, Order, Family, Species), and non-coding probability score (min: 0.8; max: 137 1.0), while 'Advanced Search' provides enhanced query functionality using logical operators 
$138(\mathrm{AND} / \mathrm{OR} />=/<=)$. Moreover, the 'Advanced Search' feature helps the users to search and apply

139 filters to query a wide range of features and metadata fields e.g. Species, Tissue, lncRNA length, 140 AlnC ID, and Non-coding probability. Consequently, a list of potential lncRNAs can be displayed 141 at the result page according to the user defined queries, with the options to download and save the results. The search results shows the entries of the chosen species, covering the basic meta details of the $1 \mathrm{kp}$ sample code with links describing the sample information, including sample preparation, sample supplier, sample extractor, tissue type NCBI ID showing experimental sample library and run data, source transcript ID from which lncRNA is annotated, lncRNA length, and non-coding probability score (Fig 3(B)). The users can further explore the AlnC ID link to view and navigate at the detailed record of the lncRNA with primary lncRNA features, secondary structure, and other useful information e.g. 'IncRNA Information'.

Fig 3 (A) Interface of simple search and advanced search modules, (B) Results page showing a table view of all lncRNA entries of the plant species Aextoxicon punctatum.

Each 'IncRNA Information' page of AlnC enables the user to access to basic information of selected lncRNA with the information of structure (Fig 3(C)). This detail page is divided into two parts; the first section contains basic details of the lncRNA sequence (including the species name of the annotated lncRNA, the source transcript ID, length, GC content and coding/non-probability), and details of the secondary structure in dot-bracket notation with a provision for the user to view and download the structures in PNG or PS format. At the second section, user can also explore the ORFs and conceptual translation products of lncRNA sequence. All the associated annotations of the concerned lncRNA entry can be downloaded in a tabular form.

160 Fig 3. (C) Sequence features including primary sequence information, secondary structure and possible sORFs as well peptides are displayed for lncRNA entry AlnC_6143890.

\section{BLAST module}

164 BLAST feature is very helpful to find regions of similarity between the user provided input 
167 Family, and Species. We have categorise the lncRNAs accordingly to avoid the memory intensive

168 search against the full dataset available at AlnC. The BLAST search output includes pairwise

169 alignment and a report with BLAST hits based upon alignment scores and other measures of

170 statistical significance. After the BLAST run, user can directly go at the entry of any particular

171 lncRNA showing similarly against the query sequence by a clink on the AlnC ID present in the

172 BLAST results.

\section{LncRNA vs mRNA module}

174 This module of $\mathrm{AlnC}$ database allows the users to visualize and compare length-wise distribution, and AU\% in annotated lncRNA transcripts and protein-coding transcripts in multiple samples of each species available at this database (Fig 3(D) and 3(E)). This page also enables the user to download the images of this comparative analysis in multiple file formats.

Fig 3. (D) Percentage composition of AU content lncRNAs relative to mRNAs, (E) Pie chart representation of length-wise distribution of Aextoxicon punctatum lncRNAs and mRNAs.

A this module, user can select the lncRNAs sequences for a particular Clade, Order, Family and Species, and download the complete sets from the AlnC archive. Complete AlnC collection can be

184 found on the download module. Here users have access to both the hierarchical bulk download and 185 the species-wise download in the FASTA file format.

\section{Submit Data}

As there are sizeable researchers working on several flowering plants, we created a user form to

190 and publications can be submitted using the form provided on the 'Submit data' page

191 (http://nipgr.ac.in/AlnC/submit_data.php) of AlnC. If the submitted data found to be relevant, the received data will be curated manually and appended to AlnC. All submitted lncRNA data will be 
curated. We encourage submissions to AlnC curators as this will drive our plans to include additional species in the future.

\section{Conclusion and future prospects}

197 In this research work, we have used the transcriptome data of 682 flowering plants, most of which had no genomic information and/or no documented lncRNA studies prior to this work. In our opinion, this is a key feature of $\mathrm{AlnC}$, which was constructed with the primary objective of promoting the investigation of lncRNA in various Angiosperm species. The analysis workflow used in this study was optimized for plants and could be used for RNA-seq-based lncRNA identification

202 for any other plant species as well. The workflow includes a ML based bioinformatics pipeline to identify high confident lncRNAs across distinct Angiosperm species, which significantly differs organised and catalogued in the AlnC web interface. It stores information of 10,855,598 lncRNA

207 loci derived from 809 samples and provides a user-friendly platform for browsing, searching and accessing all annotated lncRNAs through simple and interactive web pages. AlnC includes IncRNAs with evidence of non-coding RNA probability score, and allows further exploration of sORFs alongside other primary lncRNA features, thus providing researchers with functional

211 capability to leverage on $\mathrm{AlnC}$ data, and information on their individual projects using our web

212 interface. With this research work, we have attempted to develop a first-ever database covering the 213 largest number of confident lncRNA entries of wide-range plant species, including those with no 214 information on lncRNA of any sort. Although, it is clear that several plants belonging to 215 Angiosperms are still to be discovered and transcriptomes are waiting to be studied by individual 216 research groups, AlnC will be continuously updated. At the same time, AlnC will strive to 217 periodically search freely accessible databases, and other forms of documentation to collect useful 218 information for annotated lncRNAs, and add additional functionality to enhance user engagement. It 219 is our intention that $\mathrm{AlnC}$ will move forward to provide new databases representing additional 
220 species as well as to fine-tune, and optimise the currently available annotations. We will also aim to

221 focus on the new pipeline to develop lncRNA annotations as the plant lncRNA biology research

222 progresses. All in all, AlnC will strive to continue to be in line with the lncRNA community, and

223 remain to serve useful lncRNA data in future.

224 Conflict of interest

225 The authors declare that there is no conflict of interest.

226 Acknowledgement

227 The authors are thankful to the Department of Biotechnology (DBT)-eLibrary Consortium, India,

228 for providing access to e-resources. A.S and A.T.V are thankful to the Council of Scientific and 229 Industrial Research and the DBT for research fellowships, respectively. Also, the authors 230 acknowledge the Distributed Information Sub-Centers of DBT at the National Institute of Plant 231 Genome Research (NIPGR).

232 Author Contributions

233 A.S. and AT V. analysed the data and developed the web interface of this database. A.S., AT V., 234 and SK wrote the manuscript. S.K. conceived the idea and coordinated the project. S.K. agrees to 235 serve as the author responsible for contact and ensures communication. 


\section{References}

245 1. Thorne RF. How many species of seed plants are there? Taxon. 2002;51: 511-512. doi:10.2307/1554864

2. Christenhusz MJM, Byng JW. The number of known plants species in the world and its annual increase. Phytotaxa. 2016;261: 201-217. doi:10.11646/phytotaxa.261.3.1

3. Chen L, Zhu QH, Kaufmann K. Long non-coding RNAs in plants: emerging modulators of gene activity in development and stress responses. Planta. Springer Science and Business Media Deutschland GmbH; 2020. p. 92. doi:10.1007/s00425-020-03480-5

4. Chekanova JA. Long non-coding RNAs and their functions in plants. Current Opinion in Plant Biology. Elsevier Ltd; 2015. pp. 207-216. doi:10.1016/j.pbi.2015.08.003

5. Yu Y, Zhang Y, Chen X, Chen Y. Plant noncoding RNAs: Hidden players in development and stress responses. Annual Review of Cell and Developmental Biology. Annual Reviews

6. Cao H, Wahlestedt C, Kapranov P. Strategies to Annotate and Characterize Long Noncoding doi:10.1016/j.tig.2018.06.002 experimentally identified lncRNAs in plants. Gene. 2015;573: 328-332. doi:10.1016/j.gene.2015.07.069

9. Szcześniak MW, Bryzghalov O, Ciomborowska-Basheer J, Makałowska I. CANTATAdb 
10. Gallart AP, Pulido AH, De Lagrán IAM, Sanseverino W, Cigliano RA. GREENC: A Wikibased database of plant IncRNAs. Nucleic Acids Res. 2016;44: D1161-D1166. doi:10.1093/nar/gkv1215

11. Jin J, Lu P, Xu Y, Li Z, Yu S, Liu J, et al. PLncDB V2.0: a comprehensive encyclopedia of plant long noncoding RNAs. Nucleic Acids Res. 2020 [cited 10 Dec 2020]. doi:10.1093/nar/gkaa910

12. Cheng Quek X, Thomson DW, Maag JL V, Bartonicek N, Signal B, Clark MB, et al. lncRNAdb v2.0: expanding the reference database for functional long noncoding RNAs. Nucleic Acids Res. 2015;43. doi:10.1093/nar/gku988

13. Ayachit G, Shaikh I, Sharma P, Jani B, Shukla L, Sharma P, et al. De novo transcriptome of Gymnema sylvestre identified putative lncRNA and genes regulating terpenoid biosynthesis pathway. Sci Rep. 2019;9: 1-13. doi:10.1038/s41598-019-51355-х

14. Li A, Zhang J, Zhou Z. PLEK: A tool for predicting long non-coding RNAs and messenger RNAs based on an improved k-mer scheme. BMC Bioinformatics. 2014;15: 311. doi:10.1186/1471-2105-15-311

15. Yotsukura S, du Verle D, Hancock T, Natsume-Kitatani Y, Mamitsuka H. Computational

16. Vivek AT, Kumar S. Computational methods for annotation of plant regulatory non-coding RNAs using RNA-seq. Brief Bioinform. 2020;2020: 1-24. doi:10.1093/bib/bbaa322

17. Carpenter EJ, Matasci N, Ayyampalayam S, Wu S, Sun J, Yu J, et al. Access to RNAsequencing data from 1,173 plant species: The 1000 Plant transcriptomes initiative (1KP). Gigascience. 2019;8: 1-7. doi:10.1093/gigascience/giz126

291 18. Singh U, Khemka N, Singh Rajkumar M, Garg R, Jain M. PLncPRO for prediction of long 
non-coding RNAs (lncRNAs) in plants and its application for discovery of abiotic stressresponsive lncRNAs in rice and chickpea. Nucleic Acids Res. 2017;45: 183. doi:10.1093/nar/gkx866

19. Wickett NJ, Mirarab S, Nguyen N, Warnow T, Carpenter E, Matasci N, et al.

Phylotranscriptomic analysis of the origin and early diversification of land plants. Proc Natl

20. Consortium TU. UniProt: a worldwide hub of protein knowledge. Nucleic Acids Res. 2019;47: D506-D515. doi:10.1093/NAR/GKY1049 Acad Sci U S A. 2014;111: E4859-E4868. doi:10.1073/pnas.1323926111

21. Rombel IT, Sykes KF, Rayner S, Johnston SA. ORF-FINDER: A vector for high-throughput gene identification. Gene. 2002;282: 33-41. doi:10.1016/S0378-1119(01)00819-8

22. Altschul SF, Gish W, Miller W, Myers EW, Lipman DJ. Basic local alignment search tool. J Mol Biol. 1990;215: 403-410. doi:10.1016/S0022-2836(05)80360-2

23. Lorenz R, Bernhart SH, Höner zu Siederdissen C, Tafer H, Flamm C, Stadler PF, et al. ViennaRNA Package 2.0. Algorithms Mol Biol. 2011;6: 26. doi:10.1186/1748-7188-6-26

24. Zhao Y, Li H, Fang S, Kang Y, Wu W, Hao Y, et al. NONCODE 2016: An informative and valuable data source of long non-coding RNAs. Nucleic Acids Res. 2016;44: D203-D208.

25. Liu J, Jung C, Xu J, Wang H, Deng S, Bernad L, et al. Genome-wide analysis uncovers regulation of long intergenic noncoding RNAs in arabidopsis. Plant Cell. 2012;24: 4333-

26. Zhang W, Han Z, Guo Q, Liu Y, Zheng Y, Wu F, et al. Identification of Maize Long NonCoding RNAs Responsive to Drought Stress. Scaria V, editor. PLoS One. 2014;9: e98958. doi:10.1371/journal.pone.0098958 
316 functional analysis identify a large number of long noncoding RNAs involved in the sexual 


\section{Figure Legends}

336 Fig 1. Overview of the IncRNAs in AlnC. (A) Systematic workflow adopted to annotate potential

337 lncRNAs of all flowering plants available from the 1KP project, (B) Length-wise distribution of

338 lncRNAs across clades, (C) Pie chart represents the percentage of lncRNA entries in AlnC, (D)

339 Percentage composition of AU content in protein-coding transcripts and lncRNAs in AlnC.

340 Fig 2. Ternary plot of AlnC IncRNAs, protein-coding and 1Kp assembled transcripts across

341 clades. The bubble size represents the size of $1 \mathrm{Kp}$ assembled transcripts, protein-coding and AlnC

342 lncRNAs in (A), (B) and (C), respectively.

343 Fig 3. Screenshots of AlnC web interface. (A) Interface of simple search and advanced search

344 modules, (B) Results page showing a table view of all lncRNA entries of the plant species

345 Aextoxicon punctatum, (C) Sequence features including primary sequence information, secondary 346 structure and possible sORFs as well peptides are displayed for lncRNA entry AlnC_6143890, (D)

347 Percentage composition of AU content lncRNAs relative to mRNAs, (E) Pie chart representation of 348 length-wise distribution of Aextoxicon punctatum lncRNAs and mRNAs.

351 Table 1: Summary of annotated lncRNAs across higher-level clades in AlnC database.

\begin{tabular}{|l|l|l|l|}
\hline Clade & No. of Species & Number of Samples & Number of IncRNAs \\
\hline Basal Eudicots & 33 & 55 & 835185 \\
\hline Basalmost angiosperms & 8 & 8 & 281514 \\
\hline Chloranthales & 2 & 2 & 46074 \\
\hline Core Eudicots & 95 & 116 & 1828555 \\
\hline Core Eudicots/Asterids & 217 & 242 & 3656812 \\
\hline Core Eudicots/Rosids & 201 & 250 & 2627218 \\
\hline
\end{tabular}


bioRxiv preprint doi: https://doi.org/10.1101/2021.02.04.429715; this version posted February 5,2021 . The copyright holder for this preprint (which was not certified by peer review) is the author/funder, who has granted bioRxiv a license to display the preprint in perpetuity. It is made available under aCC-BY 4.0 International license.

\begin{tabular}{|l|l|l|l|}
\hline Magnoliids & 26 & 27 & 383010 \\
\hline Monocots & 61 & 64 & 829121 \\
\hline Monocots/Commelinids & 39 & 45 & 568109 \\
\hline Total & 682 & 809 & 10855598 \\
\hline
\end{tabular}

\section{Supporting information}

Table S1: List of significant hits of $\operatorname{lncRNAs}$ at AlnC, to lncRNAs available at NONCODE and 356 PlncDB databases. 
A Coding

1000 plant (1KP) project

\section{transcripts}

Assembled

transcripts

$\longrightarrow \begin{gathered}\text { Filtered } \\ \text { transcripts }\end{gathered} \begin{gathered}\text { ML based } \\ \text { IncRNA } \\ \text { annotation } \\ \text { (Singh et al., } \\ \text { 2017) }\end{gathered}$

High Confident

IncRNA

transcripts

bioRxiv preprint doi: https://doi.org/10.1101/2021.02.04.429715; this version posted February 5, 2021. The copyright holder for this preprint (which was not certified by peer review) is the author/funder, who has granted bioRxiv a license to display the preprint in
perpetuity. It is made available under aCC-BY 4.0 International license.

B

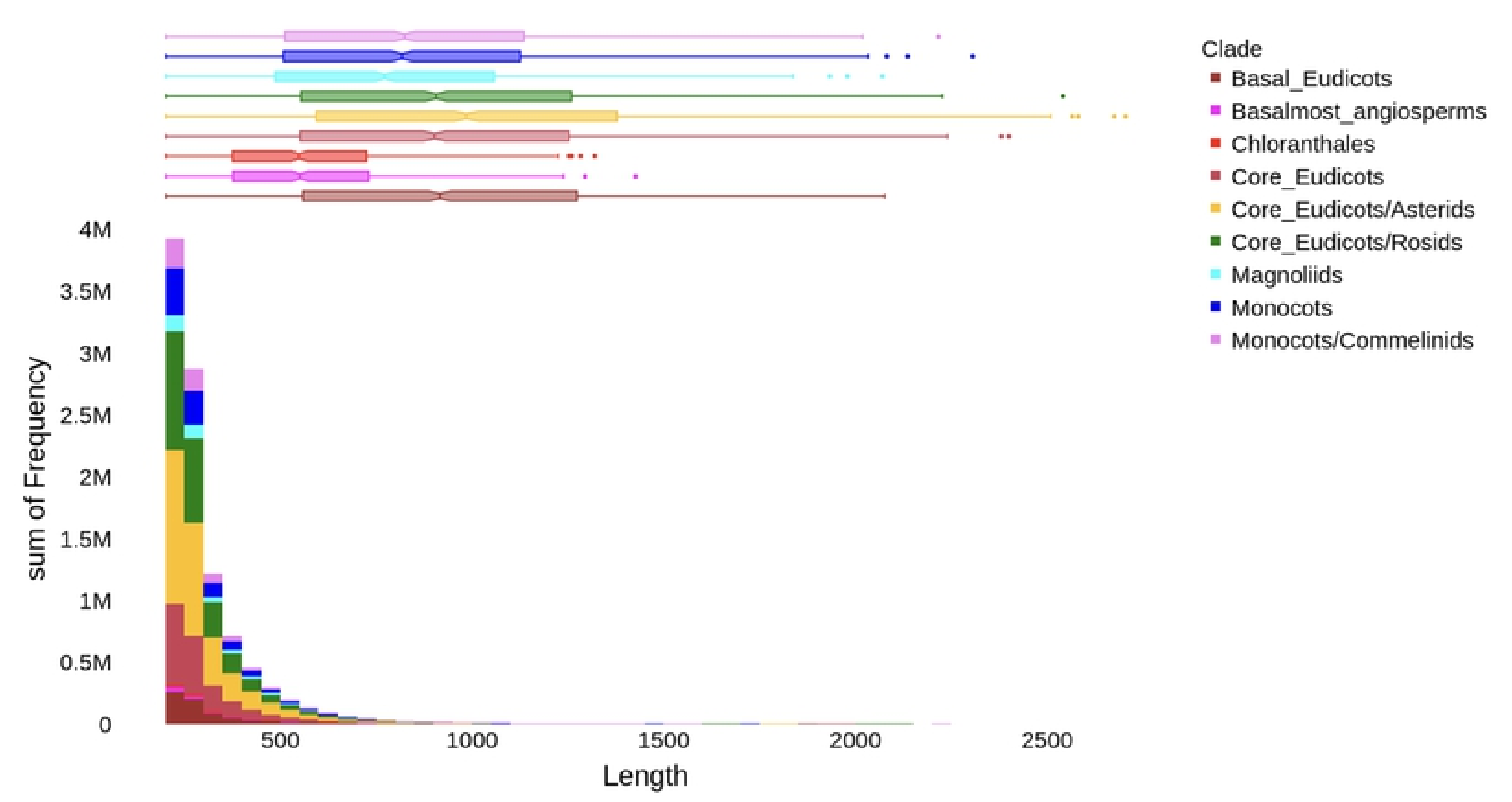

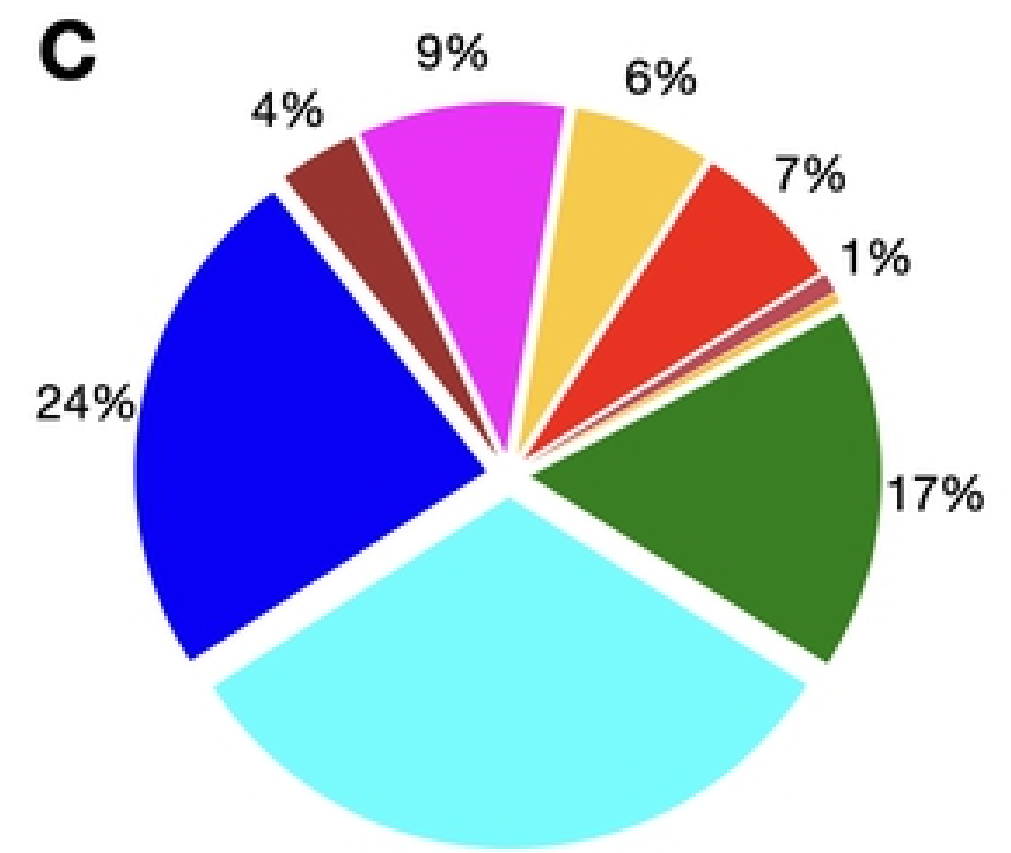

$32 \%$
- Basal_Eudicots

Basalmost_angiosperms

Chloranthales

Core Eudicots

Core_Eudicots_Asterids

Core_Eudicots_Rosids

Magnoliids

Monocots

Monocots_Commelinids

$7 \%$

Fig 1 


\section{Simple search}

\begin{tabular}{|c|c|}
\hline Seletet clume: & Bus todosos \\
\hline Meletet Corter: & 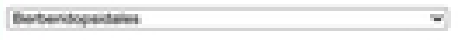 \\
\hline Solvet fangr. & netinen- \\
\hline Sulest Species: & notsonnomen \\
\hline Noneteding Mrobater. & 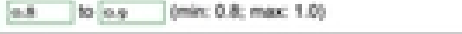 \\
\hline
\end{tabular}

\section{Advanced search}

s. mo preprint (which was' not certified by peer review) is the authorffunder, who has granted bioRxiv a license to displa s. wo preprint (which was' not certified by peer review) is the author/funder, who has granted bioRxiv a license

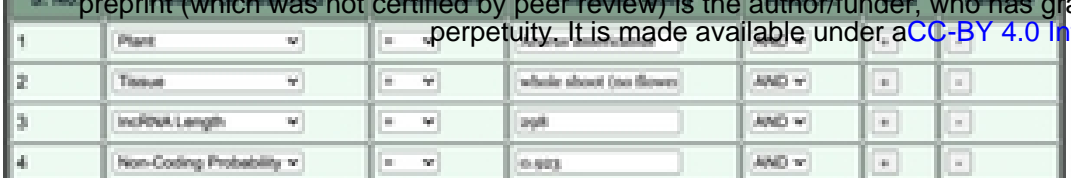

\section{C}

\begin{tabular}{|c|c|}
\hline Species & Aestoticon pusctonsm \\
\hline $\begin{array}{l}\text { Searse } \\
\text { transeript } \\
\text { ID }\end{array}$ & seaffold-QUTB-2000004-Aexioxicon_punctatum \\
\hline Sequetace & 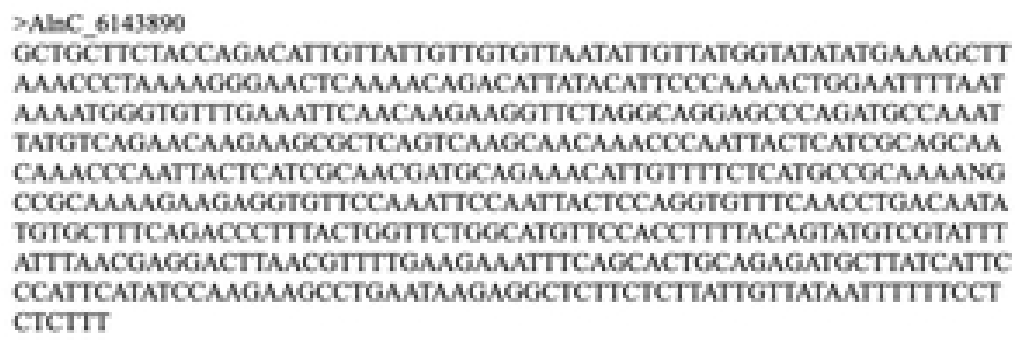 \\
\hline $\begin{array}{l}\text { Det- } \\
\text { Bracket } \\
\text { Notation }\end{array}$ & 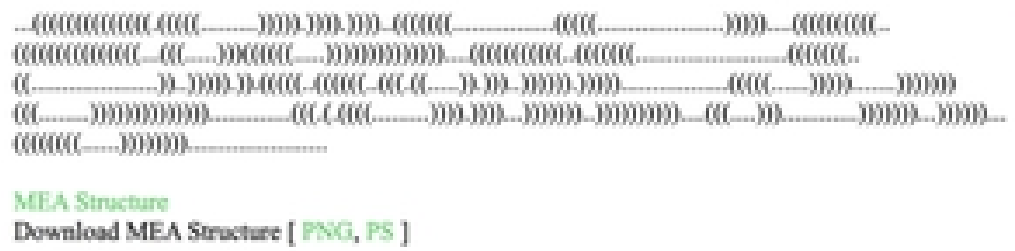 \\
\hline $\begin{array}{l}\text { Sequence } \\
\text { Length }\end{array}$ & 534 \\
\hline$G C \%$ & 0.378277 \\
\hline Probability & Non-coding 0.811 : Coding: 0.189 \\
\hline
\end{tabular}

\begin{tabular}{|c|c|c|c|c|c|c|c|}
\hline Ninc-10 & Sencien & Thave & $\begin{array}{l}\text { Sample } \\
\text { cose }\end{array}$ & $\begin{array}{l}\text { NCtal } \\
\text { ind }\end{array}$ & Source trameript iD & Lengeth & $\begin{array}{l}\text { Non-cosling } \\
\text { probutility }\end{array}$ \\
\hline$\frac{\operatorname{Aun}(6,690}{890}$ & $\begin{array}{l}\text { Aextorkicon punct } \\
\text { atum }\end{array}$ & leaves & QUTB & $\begin{array}{l}\text { ERS1L29 } \\
250\end{array}$ & 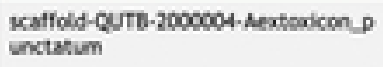 & 534 & 0.811 \\
\hline $\begin{array}{l}\text { Anc_.6143 } \\
\text { sin }\end{array}$ & $\begin{array}{l}\text { Aextouscon punct } \\
\text { atum }\end{array}$ & leaves & QUTाB & $\begin{array}{l}\text { ERS1829 } \\
250\end{array}$ & 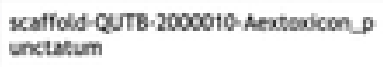 & 309 & ans \\
\hline $\begin{array}{l}\text { Anc } 6143 \\
\text { sis }\end{array}$ & $\begin{array}{l}\text { Mextoricon punkt } \\
\text { atum }\end{array}$ & leaves & QUTB & $\begin{array}{l}\text { ESS1820 } \\
250\end{array}$ & $\begin{array}{l}\text { rcatfold-QUTB-2000011. Mextexicen D } \\
\text { untatum }\end{array}$ & 412 & a.ses \\
\hline \multicolumn{2}{|c|}{$\begin{array}{l}\text { copyright holder for this, } \\
\text { display the preprint in }\end{array}$} & leswes & QUTB & $\begin{array}{l}\text { tossiass } \\
230\end{array}$ & 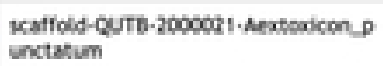 & $2 n$ & 0.838 \\
\hline $\begin{array}{l}\text { Aunc } 6143 \\
\text { Bn }\end{array}$ & $\begin{array}{l}\text { Aextowicon punct } \\
\text { atum }\end{array}$ & leaves & QuT: & $\begin{array}{l}\text { ERS1829 } \\
250\end{array}$ & $\begin{array}{l}\text { scatfold QUTB-2000002, Aextowicon P } \\
\text { unctatum }\end{array}$ & 711 & 0.092 \\
\hline $\begin{array}{l}\text { Anc } 6143 \\
\text { ans }\end{array}$ & $\begin{array}{l}\text { Aextoustion punkt } \\
\text { atum }\end{array}$ & lesves & QUTE & $\begin{array}{l}\text { Est } 1825 \\
250\end{array}$ & 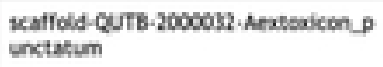 & 229 & asts \\
\hline $\begin{array}{l}\text { Anc } 6140 \\
599\end{array}$ & $\begin{array}{l}\text { Aextorkicon punct } \\
\text { atum }\end{array}$ & leaves & QUTB & $\begin{array}{l}\text { gositas } \\
250\end{array}$ & $\begin{array}{l}\text { Kcatfold-QUTB-2000034-Aextoxicon P } \\
\text { unctatum }\end{array}$ & 338 & 0.84 \\
\hline $\begin{array}{l}\text { Aunc } 6563 \\
901\end{array}$ & $\begin{array}{l}\text { Aextoricon punct } \\
\text { atum }\end{array}$ & leswes & QuTs & $\begin{array}{l}\text { ERS1829 } \\
250\end{array}$ & 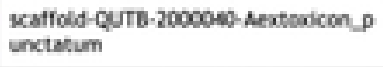 & 260 & 0.850 \\
\hline 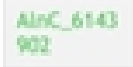 & $\begin{array}{l}\text { Aextouscon punct } \\
\text { atum }\end{array}$ & leaves & QUT: & $\begin{array}{l}\text { ERS } 1829 \\
259\end{array}$ & $\begin{array}{l}\text { reatfold-QUTB-2000050-Aextexicen_P } \\
\text { unctatum }\end{array}$ & 326 & ans \\
\hline $\begin{array}{l}\text { Anc } 6143 \\
\text { 905 }\end{array}$ & $\begin{array}{l}\text { Aextorkion punkt } \\
\text { atum }\end{array}$ & lesves & QUTB & $\begin{array}{l}\cos 1820 \\
250\end{array}$ & $\begin{array}{l}\text { Katfold-QUTB-2000077. Mextexicin D } \\
\text { unctatum }\end{array}$ & 227 & 0.891 \\
\hline
\end{tabular}

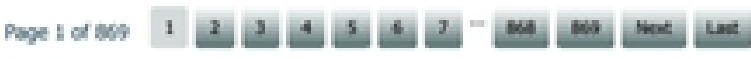

D

\section{IncRNA structure}

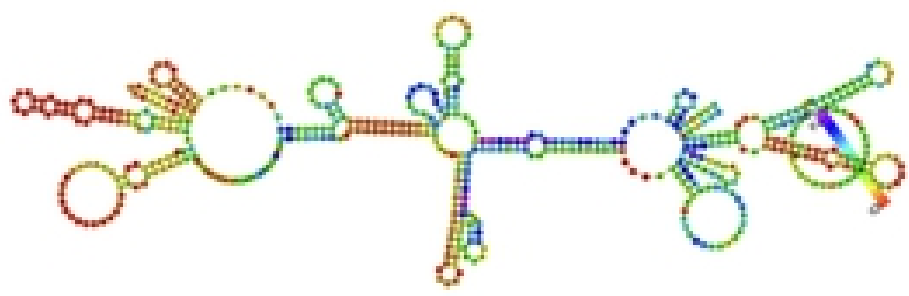

E

CDS

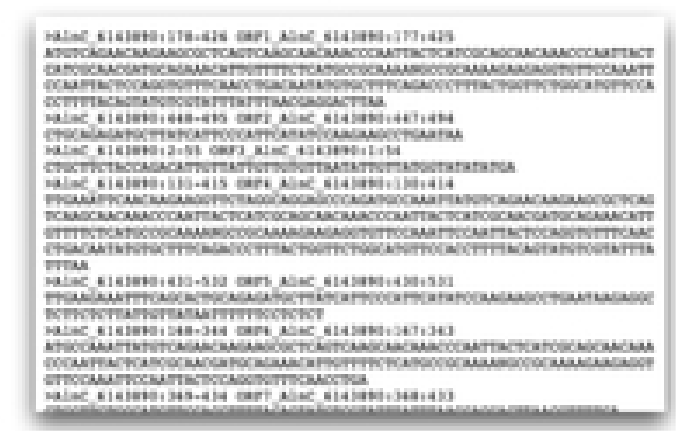

\section{Peptides}

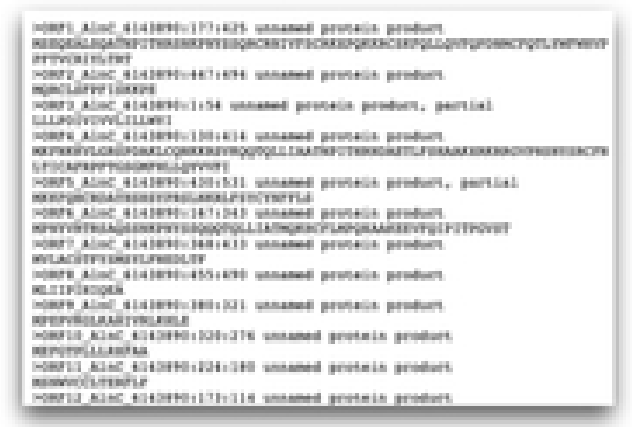

AU Content Distribution

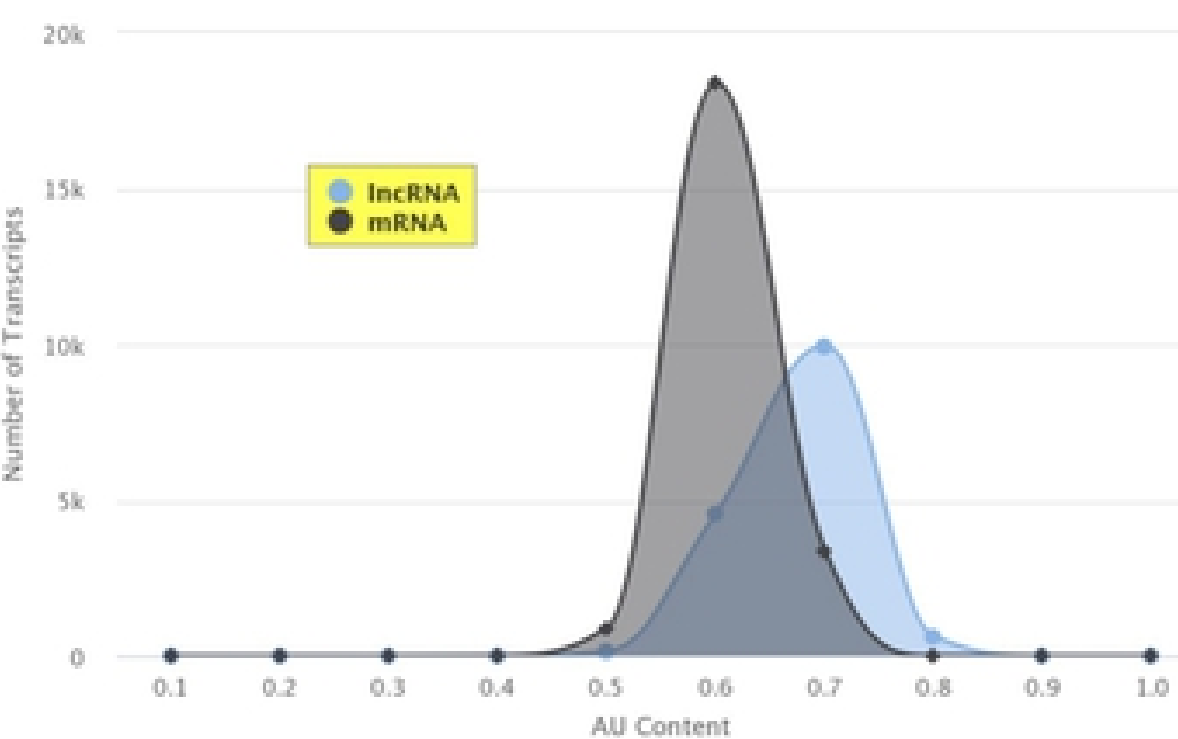

IncRNA Length Distribution

mRNA Length Distribution
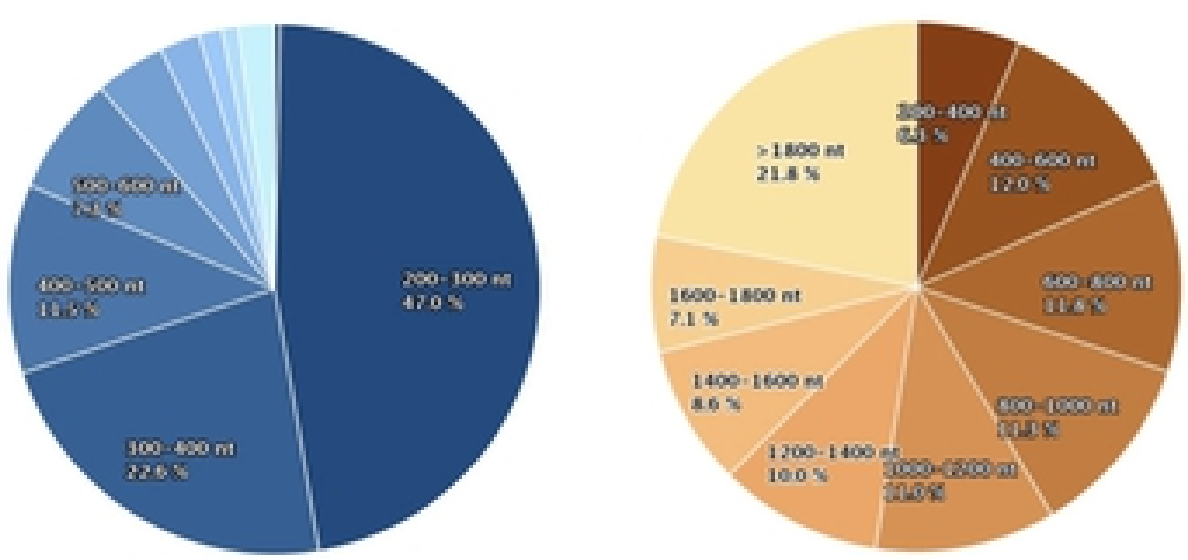

Fig 3 